\title{
Anaphylactic shock depends on PI3K and eNOS-derived NO
}

\author{
Anje Cauwels,, Ben Janssen, ${ }^{2}$ Emmanuel Buys, ${ }^{1,3}$ Patrick Sips, ${ }^{1}$ and Peter Brouckaert ${ }^{1}$ \\ ${ }^{1}$ Molecular Pathophysiology and Experimental Therapy Unit, Department for Molecular Biomedical Research, \\ Ghent University and Flanders Interuniversity Institute for Biotechnology (VIB), Ghent, Belgium. 2Department of Pharmacology and Toxicology, \\ Cardiovascular Research Institute Maastricht, University of Maastricht, Maastricht, The Netherlands. ${ }^{3}$ Cardiovascular Research Center, \\ Massachusetts General Hospital, Charlestown, Massachusetts, USA.
}

\begin{abstract}
Anaphylactic shock is a sudden, life-threatening allergic reaction associated with severe hypotension. Platelet-activating factor (PAF) is implicated in the cardiovascular dysfunctions occurring in various shock syndromes, including anaphylaxis. Excessive production of the vasodilator NO causes inflammatory hypotension and shock, and it is generally accepted that transcriptionally regulated inducible iNOS is responsible for this. Nevertheless, the contribution of NO to PAF-induced shock or anaphylactic shock is still ambiguous. We studied PAF and anaphylactic shock in conscious mice. Surprisingly, hyperacute PAF shock depended entirely on NO, produced not by inducible iNOS, but by constitutive eNOS, rapidly activated via the PI3K pathway. Soluble guanylate cyclase (sGC) is generally regarded as the principal vasorelaxing mediator of NO. Nevertheless, although methylene blue partially prevented PAF shock, neither $1 \mathrm{H}-[1,2,4]$ oxadiazole[4,3-a]quinoxalin-1-one (ODQ) nor sGC $\alpha 1$ deficiency did. Also, in 2 different models of active systemic anaphylaxis, inhibition of NOS, PI3K, or Akt or eNOS deficiency provided complete protection. In contrast to the unsubstantiated paradigm that only excessive iNOS-derived NO underlies cardiovascular collapse in shock, our data strongly support the unexpected concept that $\mathrm{eNOS}$-derived NO is the principal vasodilator in anaphylactic shock and define eNOS and/or PI3K or Akt as new potential targets for treating anaphylaxis.
\end{abstract}

\section{Introduction}

Anaphylaxis is an acute, severe, and potentially fatal systemic allergic reaction that usually attacks the patient in the absence of a history of allergy. Causes of anaphylaxis include bee stings, medications, food, latex exposure, and exercise (1). Anaphylaxis is not as rare as generally believed and may affect as much as $1.2 \%$ to $15 \%$ of the US population (2). In addition, it is generally recognized that the prevalence of anaphylaxis is increasing significantly. During an anaphylactic reaction, serious cardiovascular or pulmonary dysfunction often leads to acute death, with severe hypotension as the cardinal clinical feature. Intravenous fluids and parenteral adrenaline administration are considered the only effective treatment; unfortunately, severe cardiovascular collapse during anaphylaxis is often resistant to this treatment $(1,3)$.

Platelet-activating factor (PAF) is a biologically active phospholipid. Although PAF was originally named after its ability to induce platelet aggregation, it stimulates a broad range of cell types and tissues by binding to a unique $\mathrm{G}$ protein-coupled 7 transmembrane receptor (PAF-R), activating a number of signaling pathways, including those that elevate intracellular calcium content and activate phospholipase A2 (PLA2) and PI3K (4). As PAF antagonists or PAF-R deficiency can prevent fatal anaphylaxis in animal models, PAF is thought to be a critical factor in the development of anaphylactic shock (5-9). In addition, PAF is known to contribute to hypotension and cardiac dysfunctions during hemorrhagic, trau-

Nonstandard abbreviations used: EB, Evans blue; L-NAME, $\mathrm{N}^{\omega}$-nitro-L-arginine methyl ester; MAP, mean arterial blood pressure; MB, methylene blue; ODQ $1 \mathrm{H}-[1,2,4]$ oxadiazole[4,3-a] quinoxalin-1-one; PAF, platelet-activating factor; PLA2, phospholipase A2; sGC, soluble guanylate cyclase; WM, wortmannin.

Conflict of interest: The authors have declared that no conflict of interest exists. Citation for this article: J. Clin. Invest. 116:2244-2251 (2006). doi:10.1172/JCI25426. matic, or septic shock (4). Nevertheless, the downstream molecular pathways beyond PAF-R triggering that direct its shock-inducing effect are still enigmatic. Both ROS and NO have been implicated in PAF shock, but whether their involvement is detrimental or beneficial is still a matter of debate (10-12). However, most studies were performed in animals under general anesthesia, which influences NO-mediated effects and blood pressure considerably (13-15). Also, conflicting results in anaphylactic shock models have been reported with regard to the possible involvement of NO (16-19).

NO may be endogenously produced by the inducible iNOS or by the constitutively expressed isoforms eNOS and neuronal NOS (nNOS). While the latter are believed to produce low amounts of NO to execute physiological and/or antiinflammatory functions, the inflammation-associated expression of iNOS and the subsequent overproduction of $\mathrm{NO}$ are assumed to be responsible for the cardiovascular failure, morbidity, and mortality associated with (septic) shock $(20,21)$. In addition, it is generally accepted that NO-mediated activation of soluble guanylate cyclase (sGC) is the key mechanism in the regulation of vascular tone during (septic) shock $(22,23)$.

In this study, we aimed to unequivocally elucidate the involvement and importance of NO, sGC, ROS, and PLA2 signaling in shock induced by intravenous PAF or anaphylaxis in conscious, nonanesthetized mice. We found that PAF-induced shock completely depended on PI3K signaling and on NO produced by the constitutive enzyme eNOS and not on NO produced by the inducible "inflammatory" iNOS. Downstream from NO, PAF shock appeared to be independent of sGC. Also, in 2 different models of active systemic anaphylaxis, absolute protection was obtained by inhibition of PI3K, Akt, or NOS or by eNOS deficiency. Although it is generally accepted that excessive iNOS-derived NO underlies the cardiovascular collapse associated with shock, 

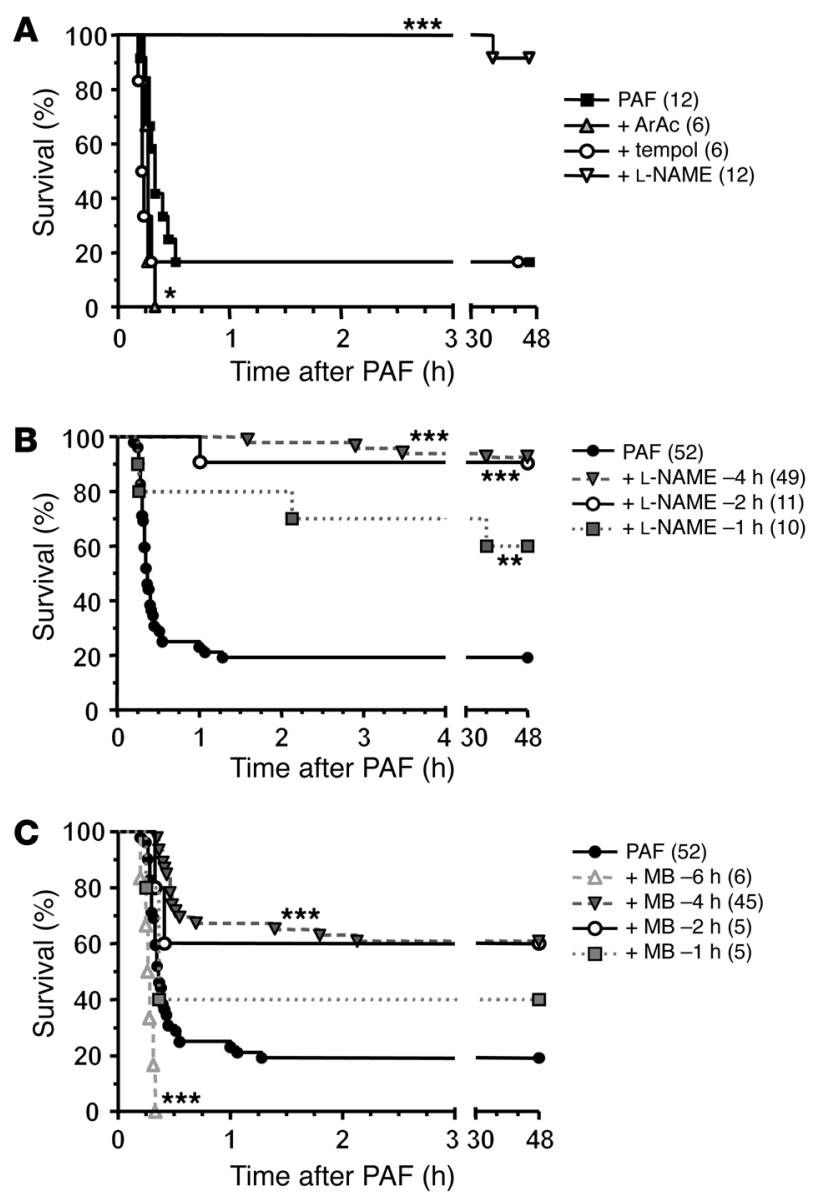

our data provide compelling new evidence for a shock-inducing function of eNOS-derived NO.

\section{Results}

NO is critical for PAF-induced shock. Intravenous PAF injection in conscious mice elicits rapid shock and results in death within 20-30 minutes (Figure 1A). When PAF engages its specific receptor, several intracellular signaling events are triggered, including PLA2 activation and superoxide production (4). Nevertheless, inhibition of these events by the PLA2 inhibitor aristolochic acid or the cellpermeable superoxide dismutase mimetic tempol did not provide any protection against PAF challenge (Figure 1A). In comparison, tempol or aristolochic acid provided complete protection against hyperacute shock induced by TNF in combination with the caspase inhibitor zVAD-fmk (24). As PAF may cause severe hypotension and iNOS induction (25), the vasorelaxant NO is generally assumed to be critically involved in PAF shock. Nonetheless, NOS inhibition cannot always preclude PAF-induced hypotension and sometimes even aggravates fatality (10-12). Since all of the aforementioned studies were performed in animals under general anesthesia, which is known to influence NO-mediated effects and blood pressure (13-15), we studied the effect of NOS inhibition on PAF hypotension and shock in conscious mice. We found that $\mathrm{N}^{\omega}$-nitro-L-arginine methyl ester (L-NAME) pretreatment completely protected against PAF lethality, implicating $\mathrm{NO}$ in a pivotal and detrimental function (Figure 1, A and B). Even when the PAF dose exceeded the $\mathrm{LD}_{100}$, L-NAME still provided absolute protection (not shown).

\section{Figure 1}

eNOS-derived NO is critical for PAF shock. C57BL/6 mice received various pretreatments and were challenged i.v. with $55 \mu \mathrm{g} P A F$; survival was monitored. (A) Pretreatment with vehicle, aristolochic acid (ArAc), tempol, or L-NAME (-4 hours). The number of mice is indicated between brackets. ${ }^{\star * \star} P<0.0001 ;{ }^{*} P=0.0201$ compared with PAF. (B) Pretreatment with L-NAME 4, 2, or 1 hour before treatment with PAF. ${ }^{* *} P=0.0165$. (C) Pretreatment with MB 6, 4, 2, or 1 hour before PAF.

$\mathrm{NO}$ is thought to relax vascular smooth muscle cells by activation of sGC and subsequent cyclic GMP-dependent modification of several intracellular processes, including the phosphorylation of proteins of the contractile apparatus and of pumps or channels involved in modulating intracellular calcium and potassium levels (26). Moreover, recent studies reported that methylene blue (MB), an inhibitor of sGC activation, can reverse clinical anaphylactic shock induced by injected contrast media and prolong survival of rabbits in experimental anaphylaxis $(27,28)$. Nevertheless, MB (15 $\mathrm{mg} / \mathrm{kg}$ ) did not prevent PAF shock to the same degree as L-NAME treatment, implying that sGC-independent $\mathrm{NO}$ activities are also involved (Figure 1, B and C). In comparison, MB provided $100 \%$ protection in a mouse model of TNF-induced shock as well as in a model of hyperacute shock induced by TNF in combination with the caspase inhibitor zVAD-fmk, as reported previously $(24,29)$. Also, lower doses of MB (5 and $10 \mathrm{mg} / \mathrm{kg}$ ) still provided partial protection (data not shown). However, pretreatment with various doses $(5,10,15$, or $20 \mathrm{mg} / \mathrm{kg})$ of another, more specific sGC inhibitor, $1 \mathrm{H}-[1,2,4]$ oxadiazole $[4,3-a]$ quinoxalin-1-one (ODQ) $(23,30)$, at different time points $(4,2$, or 0.5 hours before challenge) did not protect (data not shown). In addition, sGC $\alpha 1^{-/-}$mice (31) deficient for 1 of the 2 isoforms of the $\alpha$ subunit of the heterodimeric sGC enzyme were also not protected against PAF-induced shock (not shown). Together, these results suggest that, downstream from NO, sGC-independent events are most important.

To examine the hypotensive response to PAF, mean arterial blood pressure (MAP) was measured in unrestrained conscious mice using a catheter placed in the femoral artery. As documented before (8), intravenously administered PAF has an extremely fast and drastic hypotensive effect (Figure 2, A and C). However, when mice were pretreated with L-NAME, PAF caused only a transient and mild drop in blood pressure, followed by a very fast recovery in all but 1 of the mice (Figure 2B). All mice treated with PAF died within 13 minutes (Figure 2A) while only 1 of 5 animals pretreated with L-NAME died, after 44 minutes (Figure 2B). The MAP of the 4 surviving L-NAME-treated mice 24 hours after PAF challenge was $132 \pm 18 \mathrm{mmHg}$. We therefore conclude that $\mathrm{NO}$ plays a dominant role in acute PAF-induced hypotension. Nevertheless, other vasoactive mediators, such as histamine, serotonin, or leukotrienes, may also contribute in an NO-independent manner, albeit to a much smaller extent.

NO that causes PAF shock is produced by eNOS, activated via PI $3 K$. To identify the NOS isoform involved, we used various NOS-deficient animals. Injection of PAF in iNOS-deficient mice caused a mortality that was similar to and as rapid as that observed in WT control animals, but age-matched eNOS-deficient animals were significantly protected (Figure 3A), indicating an unexpected pivotal role for eNOS in PAF shock. Studies in vitro have shown that eNOS can be activated by direct serine phosphorylation, obviating the need for calcium and calmodulin binding and causing increased NO production $(32,33)$. This phosphorylation is mediated by the 
A

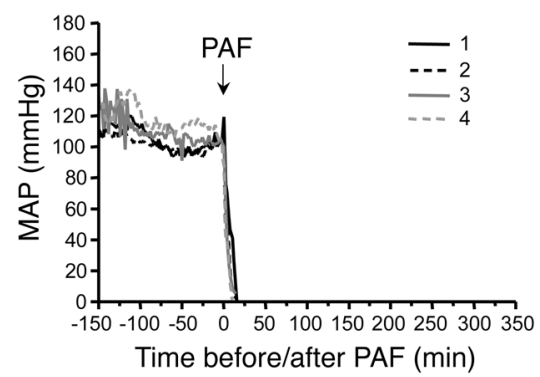

B

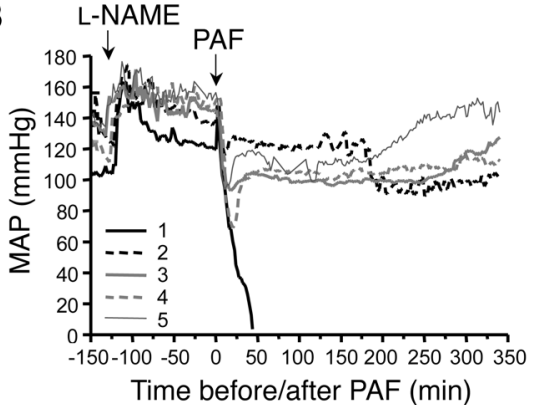

C

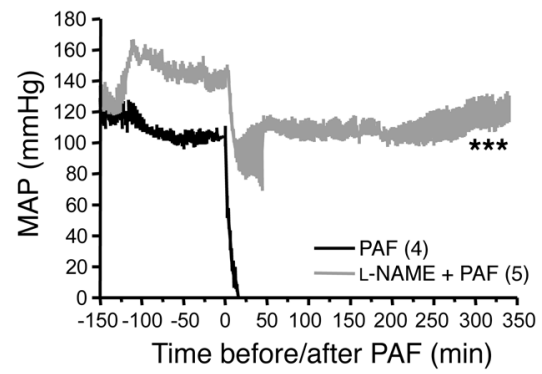

Figure 2

MAP measurements during PAF shock. C57BL/6 mice were challenged i.v. with $55 \mu \mathrm{gAF}$ at $t=0$. (A) Individual MAP of PAF-challenged mice. (B) Individual MAP of mice treated with L-NAME $(100 \mathrm{mg} / \mathrm{kg}$, i.v., -2 hours) and PAF. (C) Data points show the mean MAP \pm SD of the plots shown in $\mathbf{A}$ and $\mathbf{B}$. Error bars are plotted as vertical lines above and below the curves. The number of mice is indicated between brackets. ${ }^{* * *} P<0.0001$.

PI3K/Akt pathway, which can be activated by receptor tyrosine kinases, G protein-coupled receptors, or mechanical forces such as shear stress. Pretreatment with wortmannin (WM), a specific inhibitor of $\mathrm{PI} 3 \mathrm{~K}$, completely protected both $\mathrm{WT}$ and $\mathrm{iNOS}^{-/-}$mice (Figure 3A), demonstrating the critical importance of PI3K in PAFinduced shock. In addition, Western blot analysis of WT kidney homogenates revealed specific Ser1177 eNOS phosphorylation after PAF challenge, which can be efficiently prevented by WM pretreatment (Figure 3B).

Anaphylactic shock depends on PI3K and eNOS-derived NO. To evaluate the pathophysiological involvement of PI3K and eNOS-derived $\mathrm{NO}$ in shock in a clinically more relevant setting, we set up 2 different models of active systemic anaphylaxis, known to depend predominantly on PAF signaling $(8,9)$. Mice were first sensitized with BSA or OVA in the presence of adjuvants. A few weeks later, the animals were challenged intravenously with the same antigen. Soon after this challenge, mice developed severe hypothermia (demonstrated in Figure 4, B and D, for BSA-induced anaphylaxis) and rapidly succumbed to systemic shock reaction (Figure 4A). However, when mice were pretreated with WM or L-NAME, hypothermia was transient and less severe (Figure 4B). While 75\% of the control mice died, all 8 animals pretreated with WM or L-NAME survived the anaphylactic challenge (Figure 4A). Similar results were obtained in the OVA-induced anaphylaxis model (data not shown). Western blot analysis of WT kidney homogenates confirmed specific eNOS phosphorylation after BSA challenge in sensitized mice, prevented by WM pretreatment (Figure 4E). Next, we compared the 2 models of anaphylaxis in WT and eNOS-deficient mice. Fatality in WT animals was $82 \%$ (9 of 11 ) for the BSA model and $92 \%$ (11 of 12) for the OVA model. In contrast, none of the eNOS-deficient mice died (Figure 4C; not shown for the OVA model). Moreover, for both anaphylaxis models, there was

\section{Figure 3}

NO that causes PAF shock is produced by eNOS, activated via PI3K. (A) C57BL/6 (WT), iNOS ${ }^{-/}$, or eNOS ${ }^{-1}$ mice were injected i.v. with 55 $\mu \mathrm{g}$ PAF after pretreatment with vehicle or WM. Survival is presented as a combined Kaplan-Meier plot of 2 independent, representative experiments. The total number of mice is indicated between brackets. ${ }^{* \star \star} P<0.0001$ compared with PAF in WT mice. (B) Western blot for phosphorylated eNOS ( $p$-eNOS) in kidney homogenates of individual mice 7 or 15 minutes after PAF injection. Where indicated, mice were pretreated with WM. only a delayed, mild, and transient hypothermia in eNOS-deficient mice (Figure 4D). Neither BSA nor OVA had an effect on the temperature or behavior of unsensitized mice (Figure 4, B and D; not shown for OVA). These results reveal an unexpected but pivotal role for PI3K and eNOS-derived NO in anaphylactic shock.

During anaphylaxis, vasodilation and hypotension are often associated with excessive vascular permeability and fluid extravasation, resulting in a mixed distributive-hypovolemic shock. To assess vascular permeability, protein leakage was measured with the Evans blue (EB) technique. BSA or OVA in 1\% EB was injected into the lateral tail vein, and subsequent $\mathrm{EB}$ extravasation into the ear tissue was analyzed (Figure 4F). In the BSA/BSA anaphylactic response, vascular permeability was significantly increased in WT mice but not in $\mathrm{eNOS}^{-/-}$animals (Figure 4F). Moreover, vascular leakage in BSA/BSA eNOS ${ }^{-/}$mice was 2 -fold lower than in WT mice $(P<0.01$ by ANOVA). In OVA/OVA-induced shock, vascular permeability increased only in WT animals, and extravasation was again significantly lower in eNOS-/- OVA/OVA mice than in WT OVA/OVA animals ( $P<0.001$ by ANOVA) (not shown). In addition, pretreatment with WM or L-NAME clearly prevented EB extravasation in WT mice $(P<0.001$, data not shown). Together, these data suggest the involvement of PI3K/eNOS in vascular leakage associated with anaphylaxis.

Once activated, PI3Ks generate phosphoinositide 3,4,5-trisphosphate $\left(\mathrm{PIP}_{3}\right.$ ), leading to the recruitment and activation of Akt (protein kinase $\mathrm{B}$ ), which then activates a range of downstream targets,

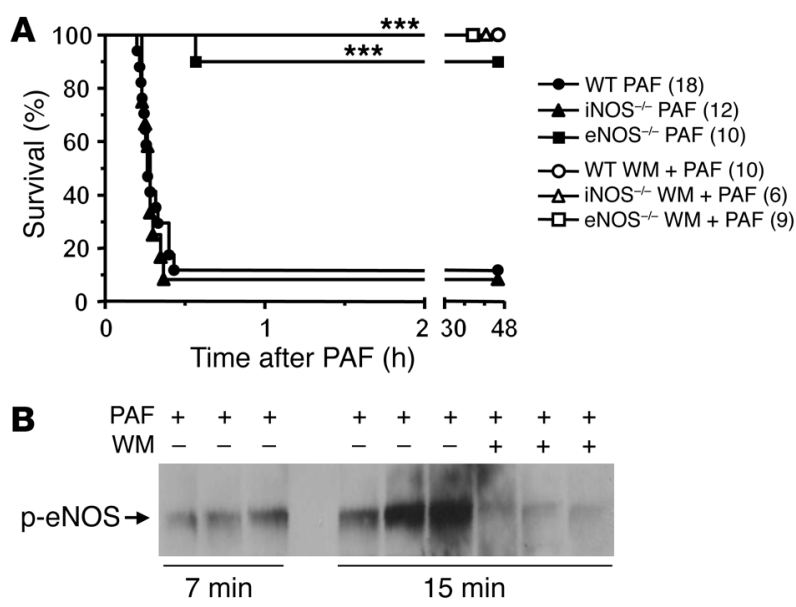


A
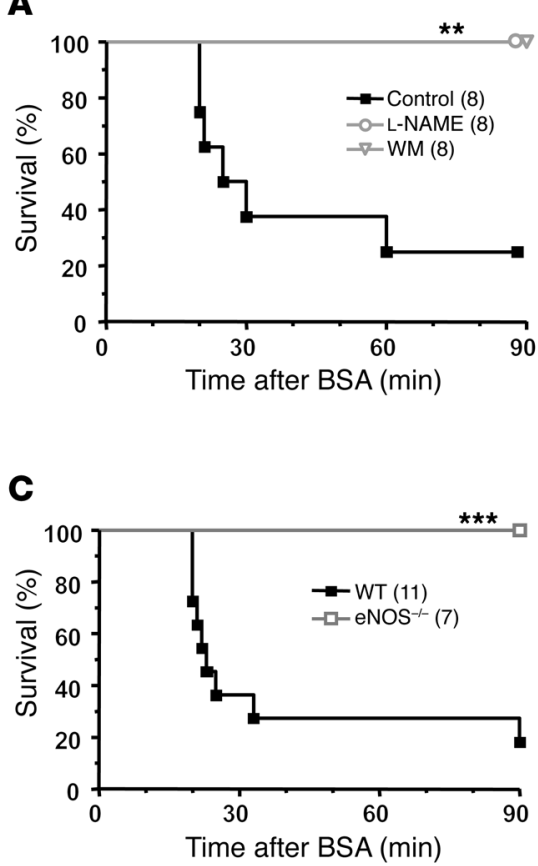

E

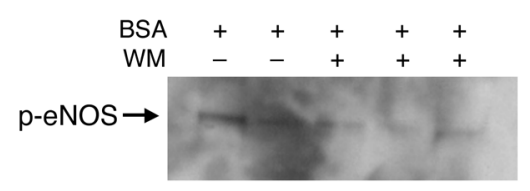

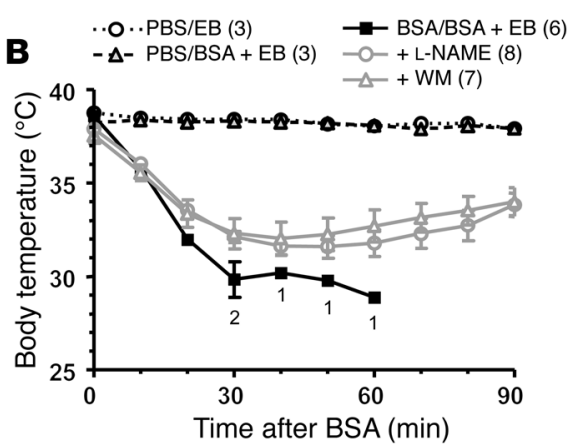
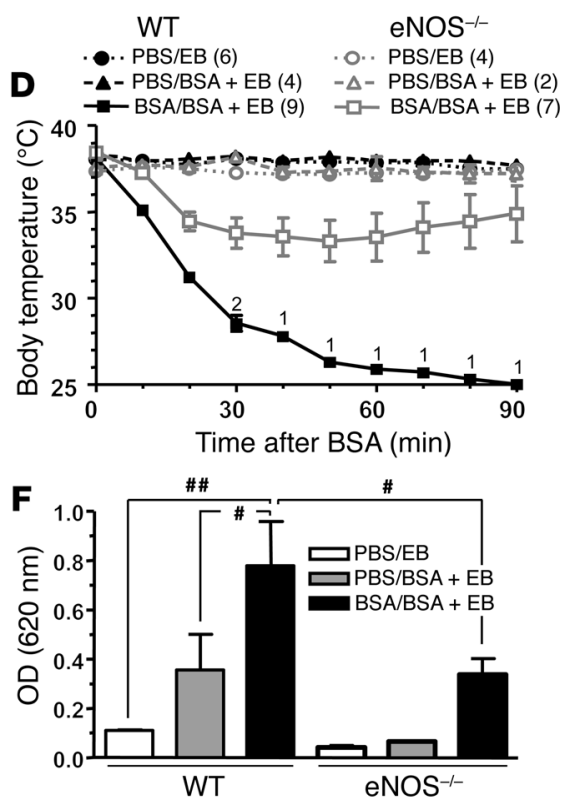

Figure 4

BSA anaphylactic shock in WT and eNOS ${ }^{-/-}$mice. (A) Survival plot of C57BL/6 mice sensitized with BSA, treated with vehicle (controls), L-NAME, or WM, and finally challenged with BSA in EB. The number of mice in each experimental group is shown in parentheses. ${ }^{\star \star} P=0.0022$. (B) Temperature plot (mean $\pm S D$ ) of mice sensitized by BSA or PBS and challenged 15 days later with $B S A$ in $E B(B S A+E B)$ or $E B$ alone. The total number of mice is shown in parentheses; numbers beneath time points indicate the number of mice still alive at the indicated times. (C) Survival plot of WT and eNOS ${ }^{-/}$mice sensitized with BSA and challenged with BSA in EB. Total number of mice is shown in parentheses. ${ }^{* *} P=0.0007$. (D) Temperature plot (mean \pm SD) of mice sensitized by BSA or PBS and challenged with BSA in EB or EB alone. The total number of mice is shown in parentheses, numbers above the time points indicate the number of mice still alive at the indicated times. (E) Western blot for p-eNOS in kidney homogenates of individual, BSA-sensitized mice 12 minutes after BSA challenge. Where indicated, mice had been pretreated with WM. (F) Vascular leakage assayed by EB extravasation into ear tissues. Inner ear rims were removed 90 minutes after i.v. challenge with $B S A$ in $E B$ or $E B$ only and subsequently extracted in formamide. Plots show mean $\mathrm{OD}_{620} \pm \mathrm{SD}$. ${ }^{\prime} P<0.001 ; \# P<0.01$.

including eNOS. Akt exists as 3 isoforms (Akt1, Akt2, and Akt3) that share extensive structural similarity and that may have unique as well as common functions within the cell. To corroborate the importance of the PI3K/Akt pathway in anaphylaxis, we treated OVA-sensitized mice with perifosine, a lipid-based inhibitor of Akt for which phase I and II clinical trials are being conducted $(34,35)$. As depicted in Figure 5 , A and B, all mice pretreated with perifosine suffered much less from hypothermia and survived the otherwise $100 \%$ lethal OVA challenge. For all Akt isoforms, knockout mice have been created (36-38). As Akt1 is the predominant Akt isoform in endothelial cells (39), we also studied anaphylactic shock in of $\mathrm{NO}$ in the cardiovascular system $(22,23,26)$.

As PAF can participate in the induction of iNOS (25), induced NO is assumed to mediate PAF-induced hypotension and shock. While some investigators have reported that NOS inhibitors can prevent PAF-induced hypotension (40, 41), others reported that it cannot $(11,12)$. However, all of these studies were performed in animals under general anesthesia, known to influence NO-mediated effects and blood pressure changes considerably (13-15). In addition, NOS inhibitors have also been reported to exacerbate PAF shock, including enhanced pulmonary hypertension (11), aggravated alveolar permeability (12), and even an increase in 

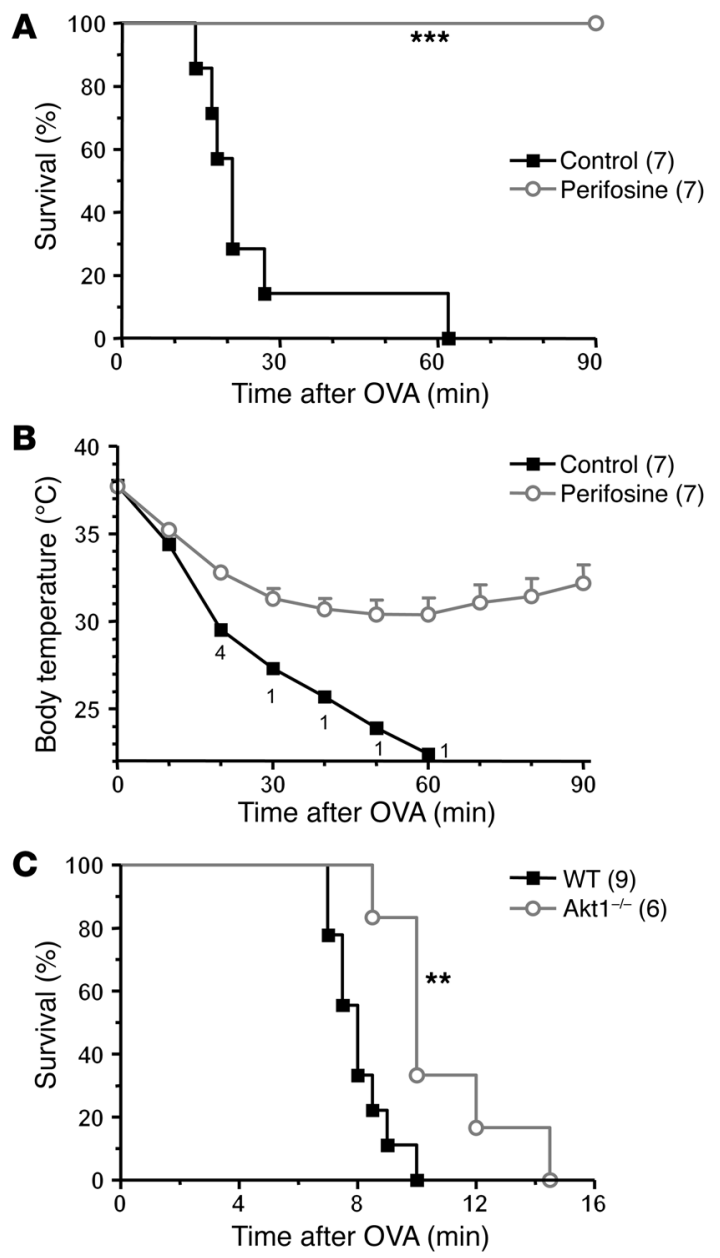

mortality of experimental animals $(10,12)$. In vitro, PAF stimulates leukocyte superoxide production, chemotaxis, and adhesion (42). Although it has been suggested that some biological activities of PAF are mediated by ROS, antioxidants such as butylated hydroxytoluene, vitamin $\mathrm{E}$, or $\mathrm{N}$-acetylcysteine failed to inhibit PAF-induced mortality, disseminated intravascular coagulation, or pulmonary edema $(43,44)$. Furthermore, the exact role of NO in various anaphylactic shock models also remains unclear, with

\section{Figure 5}

OVA anaphylactic shock in WT and Akt1 $1^{-/-}$mice. (A) Survival plot of C57BL/6 mice sensitized with OVA, pretreated with vehicle (controls) or perifosine, and challenged 19 days later with OVA in $1 \%$ EB. The total number of mice in each experimental group is shown in parentheses. ${ }^{* * *} P=0.0002$. (B) Temperature plot (mean \pm SD) of mice sensitized by OVA and challenged 19 days later with OVA in 1\% EB. The total number of mice is shown in parentheses, and numbers below the time points indicate the number of mice still alive at the indicated times. (C) Survival plot of WT and Akt1-/- mice, sensitized with OVA and challenged 20 days later with OVA in $1 \%$ EB. The total number of mice is shown in brackets in the legend. ${ }^{* *} P=0.0043$.

reports ranging from L-NAME-induced reduction of mortality (16) to reduced survival $(17,19)$.

In the present study, we set out to unequivocally elucidate the involvement and importance of NO, sGC, ROS, and PLA2 signaling in shock induced by intravenous PAF in conscious, nonanesthetized mice. To validate the results we obtained in the PAFinduced shock model, we also used 2 different models of active murine anaphylactic shock.

We found that PAF-induced mortality was not influenced by antioxidant treatment or PLA2 inhibition. Intravenous PAF injection in conscious mice elicited a very acute and drastic drop in blood pressure, resulting in death within only 20-30 minutes. As iNOS activation depends on de novo synthesis of both RNA and protein, several hours are required for its full activation. Surprisingly, however, NOS inhibition by L-NAME prevented PAF-induced hypotension and provided complete protection, even against PAF doses exceeding the $\mathrm{LD}_{100}$. Moreover, whereas PAF injection in iNOS-deficient mice caused equally rapid mortality, eNOS-deficient animals were protected, indicating an unexpected pivotal role for eNOS-derived $\mathrm{NO}$ in PAF shock. Inhibitors of sGC protected either partially (MB) or not at all (ODQ), and sGC $1^{1 /-}$ and WT mice suffered equally from PAF-induced shock. Catalytically active sGC heterodimers are composed of an $\alpha_{1}$ or $\alpha_{2}$ subunit combined with a $\beta_{1}$ subunit. Of interest, direct pharmacological sGC activation did not cause vasorelaxation or hypotension in $\mathrm{sGC} \alpha 1^{-/-}$mice, indicating that $\mathrm{sGC}$ dependent cardiovascular events predominantly rely on sGC $\alpha 1$ (31). Our results therefore imply that, downstream from NO, sGCindependent events are most important during PAF shock.

Traditionally, eNOS has been considered a constitutively expressed enzyme regulated by calcium and calmodulin. However,
A

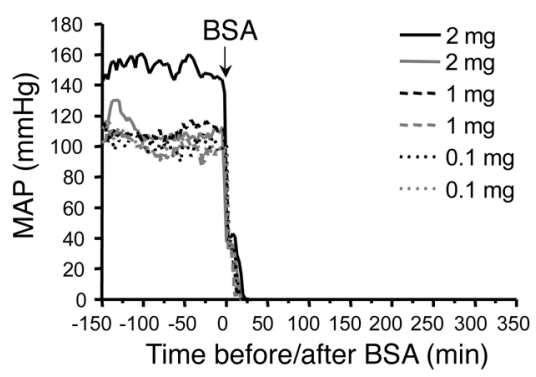

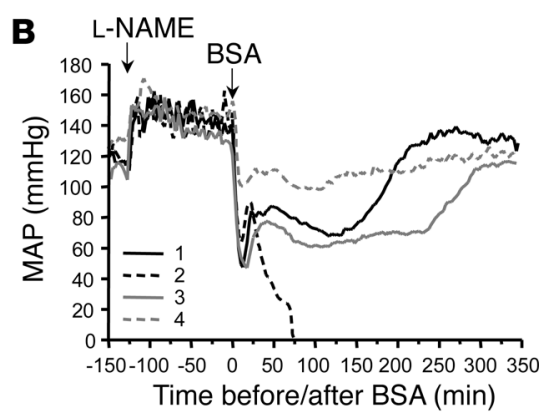

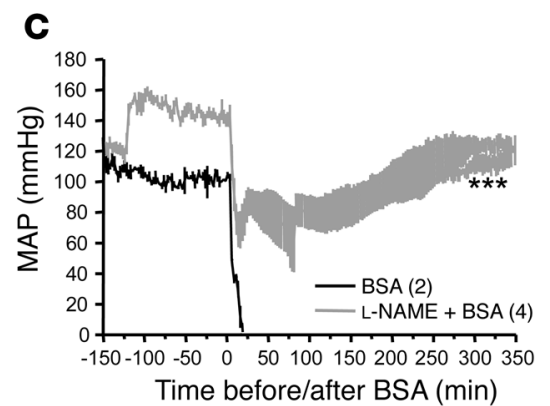

Figure 6

MAP measurements during anaphylactic shock. BSA-sensitized C57BL/6 mice were challenged i.v. with BSA at $t=0$. (A) Individual MAP of mice challenged with 2, 1, or $0.1 \mathrm{mg}$ BSA. (B) Individual MAP of mice pretreated with L-NAME $(200 \mathrm{mg} / \mathrm{kg}$, i.v.; -2 hours) and $0.1 \mathrm{mg} B S A(t=0)$. (C) Data points show the mean MAP \pm SD of the $0.1 \mathrm{mg} \mathrm{BSA}$ plots shown in $\mathbf{A}$ and $\mathbf{B}$. Error bars are plotted as vertical lines above and below the curves. Number of mice is indicated in parentheses. ${ }^{\star \star \star} P<0.0001$. 
phosphorylation of a C terminal serine residue through the PI3K/ Akt pathway activates the eNOS enzyme at resting calcium levels and/or increases its activity $(32,33,45)$. Interestingly, PI3K/Aktdependent eNOS phosphorylation and ensuing NO production occur much faster than iNOS-dependent NO production, generally reaching a maximum within only 10 to 15 minutes of stimulation $(32,46,47)$. In our study, pretreatment with WM, a specific PI3K inhibitor, prevented PAF-induced eNOS Ser1177 phosphorylation and efficiently protected both WT and iNOS-deficient mice from PAF shock. Hence, we conclude that there is a critical role for PI3K in the rapid activation of eNOS during PAF-induced shock.

To assess the pathophysiological role of PI3K/Akt and eNOSderived NO in a clinically more relevant shock setting, we tested the effects of WM, perifosine, L-NAME, and eNOS or Akt1 deficiency in 2 different active and acute systemic anaphylaxis models. The experiments revealed a critical role for PI3K/Akt and eNOSderived NO in anaphylactic shock. Following pretreatment with WM, perifosine, or L-NAME or in conditions of eNOS deficiency, anaphylaxis-induced hypothermia was mild and transient, vascular permeability was significantly lower, and no deaths occurred at all, indicating that PI3K/Akt-activated eNOS-derived NO is the most important vasodilator in anaphylactic shock.

Understanding the requirement of Akt in mammals is complicated by the existence of 3 highly conserved isoforms, Akt1, Akt2, and Akt3. Akt1-deficient mice show impaired overall growth, Akt2 knockout mice are insulin intolerant, demonstrating a diabetes-like syndrome, and mice lacking Akt3, which has more limited distribution, display a selective reduction in brain size $(36-38,48)$. Despite the protection obtained by Akt inhibition, Akt $1^{-/}$mice were not protected against anaphylactic shock, except for a minor delay in mortality. However, the viability of adult mice lacking each individual Akt isoform suggests that there is functional redundancy among these proteins. Interestingly, mice lacking both Akt1 and Akt2 die shortly after birth (49). In addition, endothelial cells of Akt1-deficient mice still show robust Akt levels and increased eNOS phosphorylation following VEGF treatment, which clearly provides evidence of the presence of compensatory mechanisms (39). This redundancy may explain why the Akt inhibitor perifosine is much more efficient than Akt 1 deficiency in preventing anaphylactic shock.

Historically, the constitutively expressed eNOS enzyme was never seriously implicated as a detrimental mediator of inflammation. On the contrary, eNOS-derived NO was generally considered to exercise a rather antiinflammatory role (20). Nevertheless, the early activation of eNOS was already linked to an early, transient vascular hyporeactivity during both endotoxic and hemorrhagic shock more than a decade ago $(50,51)$. Although eNOS was originally believed to be constitutively expressed and modulated exclusively via changes in intracellular calcium concentration, it has now become clear that eNOS can be induced several-fold by multiple stimuli (such as shear stress, estrogen, and VEGF) and posttranslationally activated through protein phosphorylation or protein-protein interactions $(45,52)$. Moreover, eNOS deficiency or eNOS inactivation efficiently prevents the acute local inflammatory response induced by subplantar carrageenan injection (53, 54). Very recently, an important function for eNOS in facilitating iNOS expression and hypotension in endotoxemia was found, demonstrating a regulatory role for eNOS-derived NO in the gene expression of inflammatory mediators such as iNOS $(55,56)$. Our present study reveals what we believe is a new and surprising con- cept that eNOS-derived NO is the principal vasodilator in PAFinduced and anaphylactic shock, thereby strongly supporting the emerging belief in a possible detrimental role for eNOS in regulating inflammation and/or vascular function during shock.

In addition, MAP measurements in anaphylactic shock demonstrated that the abrupt and precipitous drop in blood pressure, which happens immediately after the challenge and lasts only a couple of minutes, is clearly NOS/NO-independent. This may be attributed to the rapid release of preformed vasoactive mediators, such as histamine and/or serotonin. However, following this initial dramatic blood pressure drop (of $\pm 80 \mathrm{mmHg}$ ), L-NAME-pretreated mice quickly recovered and eventually survived while control animals suffered a continued steep drop in blood pressure, leading to abrupt mortality. These data clearly demonstrate that eNOS-dependent vasorelaxation is key to the sustained hypotension causing shock and mortality during anaphylaxis.

Up until lately, very little was known about the role of PI3K in the cardiovascular system. Recently, the PAF/PI3K/eNOS pathway was reported to exert a potent negative inotropic effect on the heart and to be an important cause of myocardial dysfunction following ischemia/reperfusion $(57,58)$. Our data provide important additional information on a similar detrimental effect of the PAF/ PI3K/eNOS pathway with respect to shock. In addition, we clearly demonstrate that anaphylactic shock in conscious mice is crucially orchestrated by PI3K/Akt and eNOS-derived NO. Although cyclic GMP/protein kinase G-dependent phosphorylation of the contractile machinery and calcium-regulating proteins is generally assumed to be the major mechanism by which NO causes pathological vascular smooth muscle relaxation, our results suggest that sGC-independent actions of NO may contribute significantly to its shock-inducing effect in vivo. As a general conclusion, we propose that selective targeting of eNOS and/or PI3K or Akt may provide new tools for the treatment of anaphylactic shock.

\section{Methods}

Mice. Female C57BL/6 mice were purchased from Elevage Janvier and Akt1 $1^{-/-}$ mice from Jackson Laboratory, where we also obtained iNOS-deficient (59) and eNOS-deficient (60) mice on a C57BL/6 background to breed in our facilities. The sGCo1 $1^{-/-}$mice were generated as reported (31), and littermates were used as controls. Mice were housed in temperature-controlled, air-conditioned facilities with 14-hour light/10-hour dark cycles and food and water ad libitum. All data were collected using mice 8-12 weeks of age. All experiments were approved by and performed according to the guidelines of the animal ethics committees of Ghent University, Belgium, and Maastricht University, The Netherlands.

Cytokines, reagents, injections, body temperature, and MAP measurements. PAF (1-O-alkyl-2-acetyl-sn-glycero-3-phosphocholine; Sigma-Aldrich) was diluted in $200 \mu \mathrm{l}$ endotoxin-free PBS supplemented with $0.25 \%$ BSA and injected i.v. Mortality was scored up to 7 days after challenge. The NOS inhibitor L-NAME was purchased from Novabiochem and injected i.v. at a dose of $100 \mathrm{mg} / \mathrm{kg}$. Tempol (Sigma-Aldrich) was injected i.p. at $6 \mathrm{mg} /$ mouse 1 hour before PAF. Aristolochic acid (Sigma-Aldrich) was administered s.c. in $50 \mu \mathrm{l}$ DMSO 1.5 hours before PAF at $120 \mu \mathrm{g} / \mathrm{mouse}$. MB in glucose solution suitable for i.v. injection (MB; Sterop) was used at a dose of $15 \mathrm{mg} / \mathrm{kg}$ unless otherwise stated. All of these treatments were based on the protective effects observed in our previous studies $(24,29)$. ODQ (Tocris Bioscience) was used i.p. in $50 \mu \mathrm{l}$ DMSO at $20,15,10$, or $5 \mathrm{mg} / \mathrm{kg}$. WM (Sigma-Aldrich, $30 \mu \mathrm{g} /$ mouse in 1.5\% DMSO) was given i.p. 1.3 hours before the PAF challenge. Perifosine (octadecylphosphopiperidine; Keryx Biopharmaceuticals) was injected s.c. ( $1 \mathrm{mg} / 100 \mu \mathrm{l}$ PBS/mouse) for 
4 consecutive days, the last treatment being 2 hours before challenge. Rectal body temperature was recorded with an electronic thermometer (model 2001; Comark). Blood pressure and heart rate were measured continuously in conscious, permanently catheterized C57BL/6 mice, as described (24, 29). Briefly, a heat-stretched piece of polyethylene tubing was inserted into the right femoral artery. Similarly, a catheter was placed in the jugular vein for drug injections. After surgery, animals received Ringer solution (Baxter $\mathrm{BV})$ and analgesic and were kept at $30^{\circ} \mathrm{C}$ to improve recovery. Experiments were performed 3 days after surgery, at which time the catheter was connected to a low-volume pressure transducer to record MAP and HR continuously throughout the study. The setup was allowed to stabilize for at least 40 minutes before the experiment was started.

Active systemic anaphylaxis models. Two different sensitization models were used: (a) Mice were given a single i.p. injection of $1 \mathrm{mg}$ BSA (Sigma-Aldrich) mixed with $300 \mathrm{ng}$ pertussis toxin (Sigma-Aldrich). Anaphylaxis was elicited 15 days later by i.v. injection of $2 \mathrm{mg}$ of BSA. In the hemodynamic studies, the dose of BSA had to be lowered from 2 to $0.1 \mathrm{mg}$ because the surgical procedures led to a sensitization to the BSA challenge. (b) Mice were sensitized by i.p. injection of $100 \mu \mathrm{g}$ OVA (Sigma-Aldrich), aluminum hydroxide (Sigma-Aldrich, $1 \mathrm{mg}$ ) and pertussis toxin (300 ng). Mice were challenged 19-20 days later by i.v. injection of $150 \mu \mathrm{g}$ OVA. When appropriate, mice were pretreated with L-NAME (i.v., -2.0 hours), WM (i.p., -1.3 hours), or perifosine (s.c.). Sensitizing i.p. injections were given in $0.5 \mathrm{ml}$ of endotoxin-free PBS. To permit analysis of vascular permeability, challenges were injected into the lateral tail vein in $0.2 \mathrm{ml}$ of $1 \% \mathrm{~EB}$ in endotoxin-free PBS, and ear biopsies were taken 90 minutes later. Control mice were challenged with EB only. As long as vascular permeability is unaffected, EB remains confined to the intravascular space. To quantify vascular leakage, ear biopsies were incubated in $0.3 \mathrm{ml}$ formamide at $60^{\circ} \mathrm{C}$ for 4 days to extract EB from the tissue; absorption was measured at $620 \mathrm{~nm}$.

Western blot analysis. Mice were pretreated with WM or vehicle and 1.3 hours later challenged with a lethal dose of PAF, BSA, or OVA. They were euthanized by cervical dislocation at the indicated time. Organs were dissected, snap-frozen in liquid nitrogen, and homogenized in a lysis buffer suitable for detecting phosphorylated proteins containing $50 \mathrm{mM}$ HEPES, $5 \mathrm{mM}$ EDTA, $250 \mathrm{mM} \mathrm{NaCl}, 0.1 \%$ Nonidet P-40, $0.15 \mu \mathrm{M}$ aprotinin, $2.1 \mu \mathrm{M}$ leupeptin, $1 \mathrm{mM}$ Pefabloc, $25 \mathrm{mM} \beta$-glycerolphosphate, $0.2 \mathrm{mM}$ sodium vanadate, and $10 \mathrm{mM}$ sodium fluoride. After centrifugation of cell debris, protein concentrations were determined (Bio-Rad), and $20 \mathrm{mg}$ were used for immunoprecipitation with mouse anti-eNOS (BD Biosciences - Pharmingen). Purified eNOS was resolved in a $4-12 \%$ Bis-Tris gel (Invitrogen) and transferred to nitrocellulose (Schleicher \& Schuell BioScience). Filters were blocked for 1 hour at room temperature in TBS containing $0.1 \%$ Tween 20 (TBS/T) supplemented with $5 \%$ milk powder. The filters were incubated overnight at $4^{\circ} \mathrm{C}$ with a mAb against phosphorylated eNOS (Ser1177) (Cell Signaling Technology) in TBS/T with $5 \%$ BSA, washed 5 times with TBS/T, and incubated for 1 hour at room temperature with a goat anti-rabbit IgG conjugated with horseradish peroxidase (Cell Signaling Technology). Afterwards, 5 washes with TBS/T preceded development with the Chemiluminescent Reagent Plus (ECL) detection reagent (PerkinElmer).

Statistics. Statistics were performed using GraphPad Prism version 4.0 (GraphPad Software; log-rank test for comparison of survival curves, $\chi^{2}$ test to compare total mortality percentages, unpaired 2-tailed Student's $t$ test to compare mean MAP data, and 1-way ANOVA with Bonferroni's multiple comparison test for EB extravasation data).

\section{Acknowledgments}

The authors would like to thank Linda Van Geert, Joris De Backer, and Geert Versporten for animal care; Jacques Debets and Peter Leenders for preparation of mice in the hemodynamic studies; Elke Rogge for help with immunoblotting; and Enrique Poradosu (Keryx Biopharmaceuticals) for the generous gift of perifosine. Research was supported by grants from the Fonds Wetenschappelijk Onderzoek-Vlaanderen (FWO-Vlaanderen), the Geconcerteerde Onderzoeksacties, and the Interuniversitaire Attractiepolen. A. Cauwels is a postdoctoral fellow of the FWOVlaanderen; P. Sips is an Instituut voor de aanmoediging van Innovatie door Wetenschap en Technologie fellow.

Received for publication April 21, 2005, and accepted in revised form May 2, 2006.

Address correspondence to: Anje Cauwels, Department for Molecular Biomedical Research, Technologiepark 927, B-9052 Ghent (Zwijnaarde), Belgium. Phone: 32-9-3313712; Fax: 32-9-3313609; E-mail: Anje.Cauwels@dmbr.UGent.be.
1. Kemp, S.F., and Lockey, R.F. 2002. Anaphylaxis: a review of causes and mechanisms. J. Allergy Clin. Immunol. 110:341-348.

2. Neugut, A.I., Ghatak, A.T., and Miller, R.L. 2001. Anaphylaxis in the United States: an investigation into its epidemiology. Arch. Intern. Med. 161:15-21.

3. Brown, S.G. 2005. Cardiovascular aspects of anaphylaxis: implications for treatment and diagnosis. Curr. Opin. Allergy Clin. Immunol. 5:359-364.

4. Montrucchio, G., Alloatti, G., and Camussi, G. 2000. Role of platelet-activating factor in cardiovascular pathophysiology. Physiol. Rev. 80:1669-1699.

5. Terashita, Z., Imura, Y., Nishikawa, K., and Sumida, S. 1985. Beneficial effects of (RS)-2-methoxy-3-(o ctadecylcarbamoyloxy)propyl 2-(3-thiazolio)ethyl phosphate, a specific PAF antagonist, in endotoxin and anaphylactic shock. Adv. Prostaglandin Thromboxane Leukot. Res. 15:715-717.

6. Sanchez-Crespo, M., Fernandez-Gallardo, S., Nieto, M.L., Baranes, J., and Braquet, P. 1985. Inhibition of the vascular actions of $\mathrm{IgG}$ aggregates by $\mathrm{BN}$ 52021, a highly specific antagonist of paf-acether. Immunopharmacology. 10:69-75.

7. Darius, H., Smith, J.B., and Lefer, A.M. 1986. Inhibition of the platelet activating factor mediated component of guinea pig anaphylaxis by receptor antagonists. Int. Arch. Allergy Appl. Immunol.
80:369-375.

8. Ishii, S., et al. 1998. Impaired anaphylactic responses with intact sensitivity to endotoxin in mice lacking a platelet-activating factor receptor. J. Exp. Med. 187:1779-1788.

9. Fukuda, Y., et al. 2000. Effect of human plasmatype platelet-activating factor acetylhydrolase in two anaphylactic shock models. Eur. J. Pharmacol. 390:203-207.

10. Takano, H., et al. 1997. Effects of aminoguanidine on systemic inflammatory response syndrome induced by platelet activating factor and by lipopolysaccharide in rats. Eur. J. Pharmacol. 323:107-110.

11. Wang, H.G., Shibamoto, T., Matsuda, Y., and Koyama, S. 1998. The role of endogenous nitric oxide in the sympathetic and hemodynamic response to platelet activating factor-induced hypotension in anesthetized dogs. Shock. 9:58-64.

12. Yoshikawa, T., et al. 1997. Role of superoxide and nitric oxide in platelet-activating factor-induced acute lung injury, hypotension, and mortality in rats. Crit. Care Med. 25:286-292.

13. Muldoon, S.M., Hart, J.L., Bowen, K.A., and Freas, W. 1988. Attenuation of endothelium-mediated vasodilation by halothane. Anesthesiology. 68:31-37.

14. Hart, J.L., et al. 1993. Effects of halothane on EDRF/cGMP-mediated vascular smooth muscle relaxations. Anesthesiology. 79:323-331.

15. Ma, J., et al. 1996. L-NNA-sensitive regional cerebral blood flow augmentation during hypercapnia in type III NOS mutant mice. Am. J. Physiol. 271:H1717-H1719.

16. Amir, S., and English, A.M. 1991. An inhibitor of nitric oxide production, NG-nitro-L-argininemethyl ester, improves survival in anaphylactic shock. Eur. J. Pharmacol. 203:125-127.

17. Mitsuhata, H., et al. 1995. Nitric oxide synthase inhibition is detrimental to cardiac function and promotes bronchospasm in anaphylaxis in rabbits. Shock. 4:143-148.

18. Mitsuhata, H., et al. 1995. An inhibitor of nitric oxide synthase, $\mathrm{N}$ omega-nitro-L-arginine-methyl ester, attenuates hypotension but does not improve cardiac depression in anaphylaxis in dogs. Shock. 3:447-453; discussion 454 .

19. Bellou, A., et al. 2003. Constitutive nitric oxide synthase inhibition combined with histamine and serotonin receptor blockade improves the initial ovalbumin-induced arterial hypotension but decreases the survival time in brown norway rats anaphylactic shock. Shock. 19:71-78.

20. Moncada, S. 1997. Nitric oxide in the vasculature: physiology and pathophysiology. Ann. N. Y. Acad. Sci. 811:60-67; discussion 67-69. 
21. Kirkeboen, K.A., and Strand, O.A. 1999. The role of nitric oxide in sepsis-an overview. Acta Anaesthesiol. Scand. 43:275-288.

22. Murad, F. 1996. The 1996 Albert Lasker Medical Research Awards. Signal transduction using nitric oxide and cyclic guanosine monophosphate. JAMA. 276:1189-1192.

23. Ortega Mateo, A., and Amaya Aleixandre de Artiñano, M. 2000. Nitric oxide reactivity and mechanisms involved in its biological effects. Pharmacol. Res. 42:421-427.

24. Cauwels, A., Janssen, B., Waeytens, A., Cuvelier, C. and Brouckaert, P. 2003. Caspase inhibition causes hyperacute tumor necrosis factor-induced shock via oxidative stress and phospholipase A2. Nat. Immunol. 4:387-393.

25. Szabo, C., et al. 1993. Platelet-activating factor contributes to the induction of nitric oxide synthase by bacterial lipopolysaccharide. Circ. Res. 73:991-999.

26. Lucas, K.A., et al. 2000. Guanylyl cyclases and signaling by cyclic GMP. Pharmacol. Rev. 52:375-414.

27. Oliveira Neto, A.M., Duarte, N.M., Vicente, W.V., Viaro, F., and Evora, P.R. 2003. Methylene blue: an effective treatment for contrast medium-induced anaphylaxis. Med. Sci. Monit. 9:CS102-CS106.

28. Buzato, M.A., Viaro, F., Piccinato, C.E., and Evora, P.R. 2005. The use of methylene blue in the treatment of anaphylactic shock induced by compound 48/80: experimental studies in rabbits. Shock. 23:582-587.

29. Cauwels, A., et al. 2000. Protection against TNFinduced lethal shock by soluble guanylate cyclase inhibition requires functional inducible nitric oxide synthase. Immunity. 13:223-231.

30. Zingarelli, B., Hasko, G., Salzman, A.L., and Szabo, C. 1999. Effects of a novel guanylyl cyclase inhibitor on the vascular actions of nitric oxide and peroxynitrite in immunostimulated smooth muscle cells and in endotoxic shock. Crit. Care Med. 27:1701-1707.

31. Sips, P., Buys, E., Rogge, E., Dewerchin, M., and Brouckaert, P. 2005. Functional knockout of the soluble guanylate cyclase alpha1 subunit leads to gender-specific hypertension while retaining sensitivity to nitric oxide. Circulation. 112:A747.

32. Dimmeler, S., et al. 1999. Activation of nitric oxide synthase in endothelial cells by Akt-dependent phosphorylation. Nature. 399:601-605.

33. Fulton, D., et al. 1999. Regulation of endotheliumderived nitric oxide production by the protein kinase Akt. Nature. 399:597-601.

34. Van Ummersen, L., et al. 2004. A phase I trial of perifosine (NSC 639966) on a loading dose/main- tenance dose schedule in patients with advanced cancer. Clin. Cancer Res. 10:7450-7456.

35. Granville, C.A., Memmott, R.M., Gills, J.J., and Dennis, P.A. 2006. Handicapping the race to develop inhibitors of the phosphoinositide 3-kinase/Akt/ mammalian target of rapamycin pathway. Clin. Cancer Res. 12:679-689.

36. Chen, W.S., et al. 2001. Growth retardation and increased apoptosis in mice with homozygous disruption of the Akt1 gene. Genes Dev. 15:2203-2208.

37. Cho, H., et al. 2001. Insulin resistance and a diabetes mellitus-like syndrome in mice lacking the protein kinase Akt2 (PKB beta). Science. 292:1728-1731.

38. Easton, R.M., et al. 2005. Role for Akt3/protein kinase Bgamma in attainment of normal brain size. Mol. Cell. Biol. 25:1869-1878.

39. Chen, J., et al. 2005. Akt1 regulates pathological angiogenesis, vascular maturation and permeability in vivo. Nat. Med. 11:1188-1196.

40. Takekoshi, K., et al. 1993. Effect of NG-nitro-Larginine on shock induced by endotoxin and by platelet activating factor in dogs. Eur. J. Pharmacol. 250:465-467.

41. Yamada, T., Yukioka, H., Hayashi, M., Asada, A., and Inoue, M. 1998. Effects of inhaled nitric oxide on platelet-activating factor-induced pulmonary hypertension in dogs. Acta Anaesthesiol. Scand. 42:358-368.

42. Snyder, F. 1990. Platelet-activating factor and related acetylated lipids as potent biologically active cellular mediators. Am. J. Physiol. 259:C697-C708.

43. Choi, J.H., et al. 2000. Selective involvement of reactive oxygen intermediates in platelet-activating factor-mediated activation of NF-kappaB. Inflammation. 24:385-398

44. Herbert, J.M., et al. 1993. Biochemical and pharmacological properties of SR 27388, a dual antioxidant and PAF receptor antagonist. J. Lipid. Mediat. 8:31-51.

45. Fleming, I., and Busse, R. 2003. Molecular mechanisms involved in the regulation of the endothelial nitric oxide synthase. Am. J. Physiol. Regul. Integr. Comp. Physiol. 284:R1-R12.

46. Duran, W.N., et al. 2000. Stimulation of NO production and of eNOS phosphorylation in the microcirculation in vivo. Microvasc. Res. 60:104-111.

47. Hisamoto, K., et al. 2001. Estrogen induces the Aktdependent activation of endothelial nitric-oxide synthase in vascular endothelial cells. J. Biol. Chem. 276:3459-3467.

48. Cho, H., Thorvaldsen, J.L., Chu, Q., Feng, F., and Birnbaum, M.J. 2001. Akt1/PKBalpha is required for normal growth but dispensable for mainte- nance of glucose homeostasis in mice. J. Biol. Chem. 276:38349-38352.

49. Peng, X.D., et al. 2003. Dwarfism, impaired skin development, skeletal muscle atrophy, delayed bone development, and impeded adipogenesis in mice lacking Akt1 and Akt2. Genes Dev. 17:1352-1365.

50. Szabo, C., Mitchell, J.A., Thiemermann, C., and Vane, J.R. 1993. Nitric oxide-mediated hyporeactivity to noradrenaline precedes the induction of nitric oxide synthase in endotoxin shock. Br.J.Pharmacol. 108:786-792.

51. Szabo, C. 1995. Alterations in nitric oxide production in various forms of circulatory shock. New Horiz. 3:2-32.

52. Cirino, G., Fiorucci, S., and Sessa, W.C. 2003. Endothelial nitric oxide synthase: the Cinderella of inflammation? Trends Pharmacol. Sci. 24:91-95.

53. Bucci, M., et al. 2000. In vivo delivery of the caveolin-1 scaffolding domain inhibits nitric oxide synthesis and reduces inflammation. Nat. Med. 6:1362-1367.

54. Bucci, M., et al. 2005. Endothelial nitric oxide synthase activation is critical for vascular leakage during acute inflammation in vivo. Proc. Natl. Acad. Sci. U. S. A. 102:904-908.

55. Vo, P.A., Lad, B., Tomlinson, J.A., Francis, S. and Ahluwalia, A. 2005. Autoregulatory role of endothelium-derived nitric oxide (NO) on lipopolysaccharide-induced vascular inducible NO synthase expression and function. J. Biol. Chem. 280:7236-7243.

56. Connelly, L., Madhani, M., and Hobbs, A.J. 2005. Resistance to endotoxic shock in endothelial nitricoxide synthase (eNOS) knock-out mice: a proinflammatory role for eNOS-derived $\mathrm{NO}$ in vivo. J. Biol. Chem. 280:10040-10046.

57. Alloatti, G., et al. 2003. Phosphoinositide 3-kinase gamma-deficient hearts are protected from the PAF-dependent depression of cardiac contractility. Cardiovasc. Res. 60:242-249.

58. Alloatti, G., Montrucchio, G., Lembo, G., and Hirsch, E. 2004. Phosphoinositide 3-kinase gamma: kinase-dependent and -independent activities in cardiovascular function and disease. Biochem. Soc. Trans. 32:383-386.

59. Laubach, V.E., Shesely, E.G., Smithies, O., and Sherman, P.A. 1995. Mice lacking inducible nitric oxide synthase are not resistant to lipopolysaccharide-induced death. Proc. Natl. Acad. Sci. U. S. A. 92:10688-10692.

60. Shesely, E.G., et al. 1996. Elevated blood pressures in mice lacking endothelial nitric oxide synthase. Proc. Natl. Acad. Sci. U. S. A. 93:13176-13181. 